\title{
Study on the Connection between Learning Methods of Elementary Mathematics and Advanced Mathematics
}

\author{
Bing Tang \\ Chongqing Agricultural Mechanization School, Yongchuan, Chongqing, 402160
}

Keywords: elementary mathematics; advanced mathematics; learning methods

\begin{abstract}
With the continuous reform of college entrance examinations, learning methods have become increasingly diversified. Elementary mathematics has been adjusted in teaching content, and some content overlaps with advanced mathematics. Effective teaching modes and good learning methods can enhance students in the fierce market. The core competitiveness of China, the continuous improvement of students' abilities, and the process of advanced mathematics learning methods have a certain degree of independence. It is its independence that helps students to have their own understanding and use their own advantages to actively study, constantly enhance the ability to analyze things and outline streamlined content. This paper is based on a thorough understanding of elementary mathematics teaching, targeted teaching, good transition and convergence of elementary mathematics and advanced mathematics.
\end{abstract}

\section{Introduction}

First, the introduction of the convergence of learning methods for elementary mathematics and advanced mathematics

(1) The importance of the connection between elementary mathematics and advanced mathematics learning methods

Students in different regions have different requirements for mathematics and different teaching difficulties. Therefore, teachers in classrooms should follow the cognitive rules from easy to difficult and gradual, pay attention to the connection and connection between different knowledge points, and smoothly transition to elementary mathematics. The study of advanced mathematics is easy for students to accept. In the university stage, due to the differences in previous learning methods, the gap between students' basics is large, and a large part of students' learning methods and thinking methods remain in the middle school stage. The transformation of learning methods also requires the guidance of education. Therefore, teachers Only when teaching knowledge, should we do a good job in this connection, and only in this way can students truly adapt to mathematics learning in advanced mathematics.

There is a difference between elementary mathematics and advanced mathematics. Through analysis, on the one hand, elementary mathematics deals with problems in a more intuitive manner, thus revealing a series of changes in the law of things, and analyzing and solving problems encountered in the static state. Advanced mathematics has adopted the limit means, revealing the law of changes of things through the dynamic description of the events, thus making the result more accurate. Therefore, we often use elementary mathematics to usually deal with some practical problems encountered intuitionally. On the other hand, the characteristics of higher mathematics are in stark contrast to elementary mathematics. It is the use of the ultimate means to solve more practical problems, supplementing and expanding elementary mathematics knowledge, and they are mutually reinforcing and indispensable. Therefore, the convergence of higher mathematics and elementary mathematics is necessary.

(2) The significance of the connection between elementary mathematics and advanced mathematics learning methods

In terms of teaching methods and teaching methods, elementary mathematics has the characteristics of more class hours and slower progress, and the students' dependence on instructors is very large and there is something that they do not understand. The first time they will think of 
consulting teachers. The time of independent thinking left by oneself is very few; while the higher mathematics teaching has the characteristics of more content and less class time, the student's independent learning is very important. Elementary mathematics is the foundation of advanced mathematics, and it cultivates students' logical thinking and ability to solve problems. In higher mathematics, students' autonomous learning will affect the progress and efficiency of learning. With regard to different mathematics problems, there are targeted development plans and problem-solving ideas. To control such problems, academic performance is the key to long-term, stable development. It broke the unique teaching model in the past and provided students with an open learning environment and richer resources. It has virtually cultivated students' ability to actively explore and independently think about problems. Slowly adapting to this new teaching model, it provides students with a more real-time learning platform, which has played a role in promoting the development of modern teaching.

\section{The Types of Advanced Mathematics Learning Methods}

\subsection{Shallow deep decomposition}

There are many ideas for problem solving in mathematics. There are different ways to deal with different types of problems. This is what people call targeted. The choice of method is based on the scope of application of the method and its limitations, as well as its own advantages and disadvantages. In the process of learning mathematics, before comprehensively analyzing relevant mathematics problems, we use the most commonly used methods of mathematics processing methods. The basic principles of problem solving include the following points. On the one hand, elementary mathematics will be used. The content of repetition between higher mathematics and higher mathematics is broken down according to the level of difficulty to reduce or avoid repetitions. For example, in the explanation of the sequence of numbers, elementary mathematics is only a brief introduction, while in advanced mathematics, it is extended to Computational topics, relatively in-depth and systematic discussions, through the decomposition of difficulty, also triggered students' interest in new knowledge. On the other hand, to master the most basic formulas and subtly analyze the topics, no matter how the types of topics change, they can not do without the most basic theoretical knowledge.

\subsection{Inferiority}

Take the problems we often encounter. In elementary mathematics, we learned about arithmetic progressions and geometric progressions. Regarding this concept, we will use some numerical topics to deepen the understanding of sequences. For example, let us find out the numbers. The relationship between 2, 4, 8, 16 and so on is only the simplest problem in the series. However, sometimes there are such problems, for example, one million in deposits and one in three. With interest of 30,000 yuan, the rising amount of money will then continue to be charged on the basis of the principal to collect the three-point interest. How much will it cost in ten years. What is the answer to this question? This kind of topic is quite complex at first glance. However, after serious thinking, we found that we are still using analogy equations, that is, which simple formula that we first touched, and in doing so, brought mathematics to the real life. Solving math problems in life, only in this direction, can maximize the curiosity of college students, students have curiosity, they will continue to explore new knowledge and new content, novelty and operability to students have great Appeal. The large-volume knowledge stimulates the students' interest in learning and their enthusiasm for learning improves. Only by finding a method for learning can the efficiency of learning be greatly improved.

\subsection{Comprehensive classification}

In higher mathematics, we often encounter calculus. Many students think that the problem of calculus is a difficult mathematical problem, but if we can do a comprehensive classification of each concept, we can accurately control the The origin of each formula of calculus, for example: 
$\cos x=-\sin x, \sin x d x=-\cos x+C$, there are many similar derivation formulas, these formulas require the students to accurately remember in mind, according to these formulas then Further derivation of other topics is very simple, this is the process of the calculus formula and the process of backwards, so that we will be flexible to grasp the use of this formula, the most important thing is to practice and practice, do more relevant exercises, slowly learning ideas opened, about the type of calculus problems, in the process of learning, we can comprehensively classify it, continue to learn in the classification, in the process of practice, you can add your own ideas And operations, to find more convenient and effective ways to enhance the ability to innovate, through continuous contact and solve mathematical problems, able to exercise a variety of comprehensive capabilities, which Accumulation of experience. With the development of science and technology, mathematics knowledge is updated faster and faster. Learning new knowledge and mastering effective learning methods are the primary plans. As the source of social development, it is necessary to adapt to the requirements of the development of the knowledge economy era. Study, improve their own learning ability, and thus promote their own good development.

\section{The Convergence of Learning Methods}

\subsection{Teachers teach students self-learning in teaching}

With the development of network technology, students are provided with a large amount of learning resources. For interested things, they can lie on the online teaching platform to search for information. The relevant content is on the computer screen one by one. For unsolvable math problems, for this knowledge point is not too understanding of the situation, in the Baidu search inside the relevant content, slowly students' thinking is wide, and not just stay in the simple calculations in elementary mathematics, so that the process of autonomous learning, Students are given unlimited imagination. Students continue to learn new knowledge and master new skills, develop their own potential, and in this way, they continue to improve their ability to improve the efficiency and quality of learning. When there is a goal and a direction of advancement in your own mind, you will improve your ability through continuous efforts and struggle. Your own attention is a prerequisite. When the current direction is very clear, the consciousness of learning will be very high. After students have mastered the information, they have virtually enhanced their ability to learn independently and have improved their knowledge. To achieve the exchange between teachers and classmates, students are free to express their opinions. In the process of learning, you can add your own ideas and ideas to find more convenient and effective ways to improve the students' ability to innovate.

\subsection{Teachers develop students' cooperation and communication in teaching}

The atmosphere is good and we are working as a team to solve the relationship between work-related issues. If we are in an environment where everyone has an idea and awareness of cooperation and win-win, then this atmosphere will make Those who do not know how to cooperate also feel the importance of the team. University studies do not have such a wide range of conditions. Students can study at any time. More importantly, they can freely allocate time. Students can learn as long as they have a computer. At the same time, they can learn interactively. Locally, students can use e-mail, QQ, etc. to improve their ability to think about problems. In the learning process, we must promote the spirit of this cooperation. Schools should organize regular group activities to promote interaction and exchanges among students, cultivate the spirit of mutual cooperation and mutual help among students, and communicate through the exchange of advanced mathematics. The problem. We will find ourselves in learning, which aspects are insufficient, and which knowledge points are not well understood. Through these activities, the students understand that unity is strength, a successful team, let us know the importance of cooperation and success. The team is slowly growing on the basis of cooperation. In learning, we must promote the spirit of this cooperation. For example, if there is a math problem, then the same table has helped us. However, one day, a question at the same table does not know how to calculate it. At this time, he will actively 
help him solve this difficulty. In learning, it is a group that should help each other so that they can progress together and grow together.

\subsection{Improve students' ability to solve math problems}

The teaching model is mainly divided into two parts. The first part is basic skills. In the classroom, the basic knowledge framework is first given to the students. The students can master the skills they need to master by exploring and analyzing and searching for information. The second part: technology application. The basic skills acquired are applied to practical places, and students' professional skills are improved through practice. If you only understand the basic knowledge points and do not contact them, the student's own thinking will have certain limitations. I don't know where this concept and formula will be used. For a long time, even the most basic formulas will be forgotten. To improve students' ability to solve math problems, we must constantly ask questions so that students can use the knowledge they have learned to solve practical problems. Students can experience these processes in person and use the learned knowledge flexibly. The atmosphere is conducive to the growth of students.

For example, in the education process, we must take targeted measures to guide students correctly. First, adopt a variety of teaching methods to allow students to understand and become familiar with the relevant knowledge and methods of elementary mathematics and advanced mathematics. Second, in the classroom, use innovative case teaching to inspire students' innovative ideas and broaden their horizons. Encourage students to participate in different forms of mathematics practice and organize students to participate in related competitions such as advanced math competitions. In the competition, the difficulties encountered can only be solved by themselves, and new ideas can be resolved through the use of new solutions. Problems, invisible, have increased students' sense of innovation, increased self-confidence, and increased their observational power. This is very important for future development.

\section{Conclusion}

Elementary mathematics is the basis of higher mathematics, advanced mathematics is an extension of elementary mathematics, and the two complement each other. What elementary mathematics solves are some very direct questions. Directly bringing data into the formula can yield results. However, the problems solved in higher mathematics may involve several knowledge points, and it is necessary to combine these knowledge points in order to find a solution to the problem. Due to the particularity and complexity of elementary mathematics and advanced mathematics, as well as the limited nature of my personal knowledge, I have not elaborated enough on some problems and strategies, and inevitably, I have been sparse in the writing process. Therefore, in the future, we will continue to learn as much as we can in our work and study.

\section{References}

[1] Chen Yao. Research on elementary mathematics under high perspective[J]. Good Parents, 2018

[2] Wei Yingchao. Research on the Connection between College Mathematics Education and High School Mathematics Education [J]. Examination Weekly, 2017

[3] Gan Yan. Inquiry into the convergence between advanced mathematics and elementary mathematics: taking the logarithmic function as an example [J]. Mathematical and chemical problem solving research, 2017

[4] Zhao Cuiping. Differences between elementary mathematics education and advanced mathematics education and teaching exploration [J]. Curriculum Education Research, 2015

[5] Chen Jingquan. Research on Mathematics Exam Questions in College Entrance Examination under the Background of Advanced Mathematics [J]. High School Mathematics and Physics, 2015

[6] Wang Yue. Discussion on the convergence of higher mathematics and elementary mathematics teaching in vocational colleges [J]. China Education Innovation Guide, 2013 